\title{
Deterministic Multiuser Carrier-Frequency Offset Estimation for Interleaved OFDMA Uplink
}

\author{
Zhongren Cao, Student Member, IEEE, Ufuk Tureli, Member, IEEE, and Yu-Dong Yao, Senior Member, IEEE
}

\begin{abstract}
In orthogonal frequency-division multiple access (OFDMA), closely spaced multiple subcarriers are assigned to different users for parallel signal transmission. An interleaved subcarrier-assignment scheme is preferred because it provides maximum frequency diversity and increases the capacity in frequency-selective fading channels. The subcarriers are overlapping, but orthogonal to each other such that there is no intercarrier interference (ICI). Carrier-frequency offsets (CFOs) between the transmitter and the receiver destroy the orthogonality and introduces ICI, resulting in multiple-access interference. This paper exploits the inner structure of the signals for CFO estimation in the uplink of interleaved OFDMA systems. A new uplink signal model is presented, and an estimation algorithm based on the signal structure is proposed for estimating the CFOs of all users using only one OFDMA block. Diversity schemes are also presented to improve the estimation performance. Simulation results illustrate the high accuracy and efficiency of the proposed algorithm.
\end{abstract}

Index Terms-Carrier-frequency offset (CFO), matrix decomposition, multiple access, orthogonal frequency-division multiplex (OFDM), parameter estimation.

\section{INTRODUCTION}

$\mathbf{O}$ RTHOGONAL frequency-division multiplexing (OFDM) [1] has been selected as the physical-layer technology for a number of wireless applications, such as digital audio broadcasting (DAB) [2] and terrestrial digital video broadcasting (DVB) [3]. It has also been adopted by IEEE 802.11a [4] and HIPERLAN/2 [5] for high-data-rate wireless packet transmissions. In OFDM, a set of equally spaced subcarriers is used for parallel data transmission. To increase the frequency efficiency, these subcarriers are overlapping in the frequency domain, but orthogonal to each other such that there is no intercarrier interference (ICI). The most important feature of OFDM is the capability of mitigating frequency-dependent distortion across the channel band and simplifying the equalization in a multipath fading environment.

Recently, orthogonal frequency-division multiple access (OFDMA) was proposed for broadband wireless multiple-

Paper approved by R. Reggiannini, the Editor for Synchronization and Wireless Applications of the IEEE Communications Society. Manuscript received September 25, 2002; revised December 10, 2003 and March 20, 2004. This paper was presented in part at the 36th Asilomar Conference on Signals, Systems, and Computers, Pacific Grove, CA, November 2002 and in part at the IEEE International Conference on Communications, Anchorage, AK, 2003.

Z. Cao was with the Department of Electrical and Computer Engineering, Stevens Institute of Technology, Hoboken, NJ 07030, USA. He is now with the Department of Electrical and Computer Engineering, University of California, San Diego, San Diego, CA 92037 USA (e-mail: zrcao@ece.ucsd.edu).

U. Tureli and Y.-D. Yao are with the Department of Electrical and Computer Engineering, Stevens Institute of Technology, Hoboken, NJ 07030 USA (e-mail: utureli@stevens.edu; yyao@stevens.edu).

Digital Object Identifier 10.1109/TCOMM.2004.833183 access systems in IEEE 802.16a [6]. In OFDMA, closely spaced and overlapped subcarriers are divided into groups and assigned to multiple users for simultaneous transmissions. Unlike traditional frequency-division multiple access (FDMA), where any overlapping of the frequency spectrum of different users introduces multiple-access interference (MAI), the orthogonality of subcarriers guarantees that there is no ICI, which prevents MAI among users in OFDMA systems.

OFDMA inherits from OFDM the weakness of being sensitive to inaccurate frequency references [7]. Carrier-frequency offset (CFO) between the transmitter and the receiver causes the loss of orthogonality among subcarriers and introduces ICI. In OFDMA, CFO will further cause MAI, which degrades the system performance [8]. CFO estimation for OFDM has been extensively studied in recent years [9]-[14]. In OFDMA, CFO estimation is relatively simple in the broadcast link (downlink), where different users' signals are multiplexed by the same transmitter, and the orthogonality among all subcarriers is maintained. Each user can perform the frequency synchronization by estimating a single CFO between itself and the transmitter, and compensate accordingly. Many CFO-estimation algorithms proposed for OFDM are applicable to the OFDMA downlink. The real challenge exists in the uplink of OFDMA, where a number of users share the total number of subcarriers, and each user has its own CFO. CFO estimation, in this case, becomes a multiple-parameter estimation problem.

CFO estimation in the OFDMA uplink is closely related to the subcarrier-assignment scheme adopted by the system. There are two major subcarrier-assignment schemes [8], subband based and interleaved. The former, subband-based subcarrier assignment, divides the whole bandwidth into small continuous subbands, and each user is assigned to one or several subbands, similar to traditional FDMA. In the latter, interleaved subcarrier assignment, subcarriers assigned to different users are interleaved over the whole bandwidth.

CFO estimation in the uplink of subband-based OFDMA systems has been investigated and reported recently in [15]-[17], where a frequency-guard band was used between subbands so that signals from different users can be separated by filter banks. Existing CFO-estimation algorithms that were proposed for OFDM are applied to each single user after its signal is isolated.

Subband-based OFDMA systems are vulnerable to frequency-selective fading. In channels with a large coherent bandwidth, several consecutive subcarriers may be subject to deep fading at the same time, and the data transmitted over a whole subband may not be recovered, even with coding and interleaving. On the contrary, the interleaved subcarrier- 
assignment scheme provides maximum separation among the subcarriers assigned to the same user, which maximizes the frequency diversity for each user. However, the interleaved scheme complicates CFO estimation in the OFDMA uplink, since it minimizes the distances between subcarriers assigned to different users. When there are frequency-synchronization errors, signals from different users are overlapping in the time domain by the nature of OFDMA, and interfering with each other in the frequency domain, due to the loss of orthogonality. In order to separate multiple users' signals and suppress MAI, advanced signal-processing algorithms have to be applied at the uplink receiver, as shown in [18]. These techniques rely on the correct estimation of each user's CFO. Therefore, CFO estimation is a major task in designing OFDMA receivers.

CFO estimation in the interleaved uplink of OFDMA was studied, and a statistics-based CFO-estimation algorithm was proposed for OFDMA, which is applicable to both subband-based uplink and interleaved uplink [19]. However, the algorithm in [19] requires collecting signal samples of many OFDMA blocks to compute unknown parameters on the order of the number of subcarriers, and results in a biased estimator. When the number of subcarriers is large, such as 2048 as in IEEE 802.16, the algorithm is computationally prohibitive.

The originality of this paper is that it investigates the CFO estimation in the interleaved uplink of OFDMA based on the deterministic structure of the signals. In an interleaved OFDMA system, the signal from each user has a special periodic structure within an OFDMA block, which can be exploited by arranging the received signals into a matrix form to reduce the number of unknown frequencies to the number of users. A high-resolution signal-processing technique [20] is used to estimate the CFOs of all involved users, deterministically using only one OFDMA block instead of a large number of blocks, as in [19]. Since the number of unknown parameters equals the number of users, the algorithm is practical for OFDMA systems with a large number of subcarriers.

This paper is organized as follows. The signal model of OFDMA is presented in Section II. The periodic signal structure of the interleaved OFDMA uplink is introduced in Section III. In Section IV, the CFO-estimation problem is stated and the proposed estimation algorithm is derived. Simulation results are reported in Section V, and conclusions are drawn in Section VI.

\section{OFDM-BASED MULTIPLE-ACCESS OFDMA}

In this section, we introduce the uplink signal model of OFDMA. Consider an OFDMA system consisting of $N$ subcarriers and $K$ users. The $N$ subcarriers include all available subcarriers and virtual subcarriers in the guard band [21]. All subcarriers are sequentially indexed with $\{n\}, n=0,1, \ldots, N-1$. Among the $N$ subcarriers, the $k$ th user is assigned to a subset of $P^{(k)}$ subcarriers with the index set $\left\{c_{0}^{(k)}, c_{1}^{(k)}, \ldots, c_{P^{(k)}-1}^{(k)}\right\}$. The superscript $(\cdot)^{(k)}$ denotes the $k$ th user.

An OFDMA block is the signal generated by one inverse fast Fourier transform (IFFT) operation including the cyclic prefix

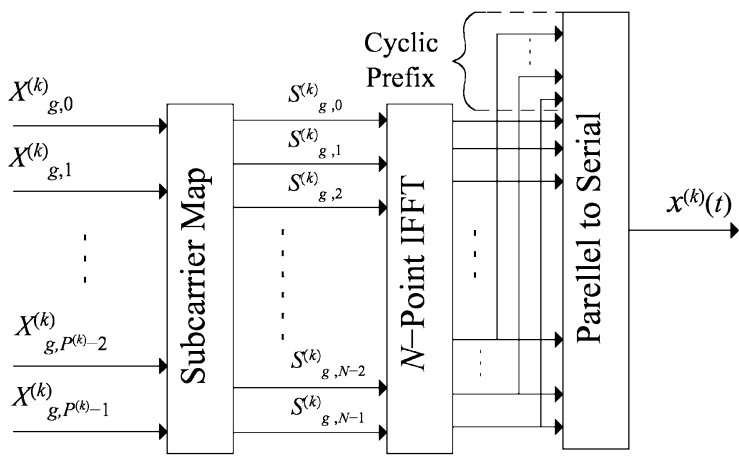

Fig. 1. Uplink OFDMA transmitter structure.

(CP). Let $\left[X_{g, 0}^{(k)}, X_{g, 1}^{(k)}, \ldots, X_{g, P^{(k)}-1}^{(k)}\right]$ be the $P^{(k)}$ modulation symbols the $k$ th user will transmit during the $g$ th OFDMA block. For data-bearing subcarriers, the modulation symbols are data symbols, such as phase-shift keying (PSK) or quadrature amplitude modulation (QAM). For virtual subcarriers, the modulation symbols are effectively padded zeros in IFFT. For pilot subcarriers, the modulation symbols are pilot symbols or training symbols for estimating the channels.

Fig. 1 illustrates the signal generation and transmission of the $k$ th user. The $P^{(k)}$ modulation symbols are first mapped into a set of $N$ modulation symbols, $S_{g, i}^{(k)}, i=0,1, \ldots, N-1$, according to

$$
S_{g, i}^{(k)}= \begin{cases}X_{g, p}^{(k)}, & \text { if } i=c_{p}^{(k)} \\ 0, & \text { otherwise }\end{cases}
$$

for $p=0,1, \ldots, P^{(k)}-1$. The $N$ symbols, $\left\{S_{g, i}^{(k)}\right\}_{i=0}^{N-1}$, are modulated onto the $N$ subcarriers via an $N$-point IFFT. The CP is also added to avoid interblock interference (IBI) caused by multipath fading. As a result, the baseband signal transmitted from the $k$ th user can be represented as

$$
\begin{aligned}
x^{(k)}(t) & =\sum_{g=-\infty}^{\infty} \sum_{i=0}^{N-1} S_{g, i}^{(k)} F_{g, i}(t) \\
& =\sum_{g=-\infty}^{\infty} \sum_{p=0}^{P^{(k)}-1} X_{g, p}^{(k)} F_{g, c_{p}^{(k)}}(t) .
\end{aligned}
$$

Note $F_{g, i}(t)$ is given by

$$
F_{g, i}(t)= \begin{cases}e^{j 2 \pi(i \Delta f)\left(t-T_{\mathrm{cp}}-g T_{b}\right)}, & g T_{b} \leq t<(g+1) T_{b} \\ 0, & \text { otherwise }\end{cases}
$$

where $\Delta f$ is the subcarrier spacing, $T_{\mathrm{cp}}$ is the length of $\mathrm{CP}$, and $T_{b}=T+T_{\mathrm{cp}}$ is the duration of one OFDMA block, $T=1 / \Delta f$.

The signals are transmitted through slowly time-variant multipath fading channels, i.e., fading coefficients are assumed to be constant during one OFDMA block. The channel between the $k$ th user and the uplink receiver is characterized by

$$
h^{(k)}(\tau, t)=\sum_{l=1}^{L^{(k)}} \alpha_{l}^{(k)}(t) \delta\left(\tau-\tau_{l}^{(k)}\right)
$$

where $L^{(k)}$ is the total number of paths, and $\alpha_{l}^{(k)}$ and $\tau_{l}^{(k)}$ are the complex gain and time delay of the $l$ th path. 
At the uplink receiver, the signal of one OFDMA block is the superposition of signals from all $K$ involved users. Assuming all $K$ users are synchronized in time, the received sampled signal in the absence of noise can be written as

$$
\Upsilon\left(n T_{s}\right)=\sum_{k=1}^{K} \sum_{l=1}^{L^{(k)}} \alpha_{l}^{(k)}\left(n T_{s}\right) x^{(k)}\left(n T_{s}-\tau_{l}^{(k)}\right)
$$

where $T_{s}=T / N$ is the sampling interval. As we will focus on the signal of one OFDMA block, the index $g$ in the following is neglected for convenience. Let $H_{p}^{(k)}$ denote the channel frequency response on the $c_{p}^{(k)}$ th subcarrier of the $k$ th user's channel during one OFDMA block. We have

$$
H_{p}^{(k)}=\sum_{l=1}^{L^{(k)}} \alpha_{l}^{(k)} e^{-j 2 \pi c_{p}^{(k)} \Delta f \tau_{l}^{(k)}}
$$

From (2)-(6), after the removal of $\mathrm{CP}$, the remaining $N$ signal samples of one OFDMA block at the uplink receiver are given by

$$
\begin{aligned}
\Upsilon(n) & =\sum_{k=0}^{K-1} \sum_{p=0}^{P^{(k)}-1} H_{p}^{(k)} X_{p}^{(k)} e^{j 2 \pi\left(c_{p}^{(k)} \Delta f\right) n T_{s}} \\
& =\sum_{k=0}^{K-1} \sum_{p=0}^{P^{(k)}-1} H_{p}^{(k)} X_{p}^{(k)} e^{j(2 \pi / N) n c_{p}^{(k)}}
\end{aligned}
$$

where $n=0,1, \ldots, N-1$.

Let $\Delta f^{(k)}$ denote the CFO between the $k$ th user and the uplink receiver. For practical purposes, the absolute value of $\Delta f^{(k)}$ is assumed to be less than the half of OFDMA subcarrier spacing. Following [14], the presence of $\left\{\Delta f^{(k)}\right\}_{k=1}^{K}$ changes the signal model of (7) to

$$
\Upsilon(n)=\sum_{k=1}^{K} \sum_{p=0}^{P^{(k)}-1} H_{p}^{(k)} X_{p}^{(k)} e^{j(2 \pi / N) n\left(c_{p}^{(k)}+\xi^{(k)}\right)}
$$

where $\xi^{(k)} \in(-0.5,0.5)$ is given by

$$
\xi^{(k)}=\frac{\Delta f^{(k)}}{\Delta f}
$$

and it is defined as the normalized $C F O$ of the $k$ th user.

\section{Signal Structure IN THE UPLinK OF INTERLEAVED OFDMA SYSTEM}

In this section, the signal structure at the interleaved OFDMA uplink is presented, which will be used for CFO estimation later. Suppose the $N$ subcarriers are divided into $Q$ subchannels, and each subchannel has $P=N / Q$ subcarriers. For interleaved subcarrier assignment, subchannel $\{q\}$ is composed of subcarriers with index set $\{q, Q+q, \ldots,(P-1) Q+q\}, q=$ $0,1, \ldots, Q-1$.

\section{A. Single-User Signal Structure and Effective CFO}

For the purpose of derivation, consider the system has only one user $k$, which is assigned to subchannel $\{q\}$. After the re- moval of $\mathrm{CP}$, the received $N$ signal samples of the OFDMA block from the $k$ th user can be written as

$$
\begin{aligned}
\Upsilon^{(k)}(n) & =\sum_{p=0}^{P-1} H_{p}^{(k)} X_{p}^{(k)} e^{j \frac{2 \pi}{N}\left(p Q+q+\xi^{(k)}\right) n} \\
& =e^{j \frac{2 \pi}{N}\left(q+\xi^{(k)}\right) n} \sum_{p=0}^{P-1} H_{p}^{(k)} X_{p}^{(k)} e^{j \frac{2 \pi}{P} p n}
\end{aligned}
$$

where $n=0,1, \ldots, N-1$. It is important to note that

$$
\Upsilon^{(k)}(n+P)=e^{j 2 \pi\left(q+\xi^{(k)}\right) / Q} \Upsilon^{(k)}(n)
$$

Furthermore, we have

$$
\Upsilon^{(k)}(n+\nu P)=e^{j 2 \pi \nu\left(q+\xi^{(k)}\right) / Q} \Upsilon^{(k)}(n)
$$

where $\nu$ is an integer. Equations (11) and (12) indicate that the received $N$ signal samples of one OFDMA block from the $k$ th user, $\left\{\Upsilon^{(k)}(n)\right\}_{n=0}^{N-1}$, have a special periodic structure with every $P$ samples. Let $R$ denote the number of periods within the $N$ signal samples. We have $R=N / P=Q$. The number of periods is the number of subchannels. $\left\{\Upsilon^{(k)}(n)\right\}_{n=0}^{N-1}$ can thus be arranged into a $R \times P$ matrix

$$
\mathbf{A}^{(k)}=\left[\begin{array}{ccc}
\Upsilon^{(k)}(0) & \ldots & \Upsilon^{(k)}(P-1) \\
\Upsilon^{(k)}(P) & \cdots & \Upsilon^{(k)}(2 P-1) \\
\vdots & \ddots & \vdots \\
\Upsilon^{(k)}(N-P) & \ldots & \Upsilon^{(k)}(N-1)
\end{array}\right]_{R \times P}
$$

And (10) can be expressed concisely as

$$
\mathbf{A}^{(k)}=\boldsymbol{v}^{(k)}\left\{\boldsymbol{u}^{(k)} \odot\left(\boldsymbol{b}^{(k)} \mathbf{W}\right)\right\}
$$

In (14), $\odot$ represents the Schur product [22], or element-byelement product. $\mathbf{W}$ is a $P \times P$ IFFT matrix given as

$$
\begin{aligned}
\mathbf{W} & =\left[\begin{array}{cccc}
1 & 1 & \cdots & 1 \\
1 & e^{j \frac{2 \pi}{P}} & \cdots & e^{\frac{2 \pi(P-1)}{P}} \\
\vdots & \vdots & \ddots & \vdots \\
1 & e^{j \frac{2 \pi(P-1)}{P}} & \cdots & e^{j \frac{2 \pi(P-1)(P-1)}{P}}
\end{array}\right] \\
\boldsymbol{b}^{(k)} & =\left[\begin{array}{llll}
H_{1}^{(k)} X_{1}^{(k)} & H_{2}^{(k)} X_{2}^{(k)} & \cdots & H_{P}^{(k)} X_{P}^{(k)}
\end{array}\right] .
\end{aligned}
$$

Also

$$
\begin{aligned}
\boldsymbol{v}^{(k)} & =\left[\begin{array}{llll}
1 & e^{j 2 \pi \theta^{(k)}} & \cdots & e^{j 2 \pi(Q-1) \theta^{(k)}}
\end{array}\right]^{\mathrm{T}} \\
\boldsymbol{u}^{(k)} & =\left[\begin{array}{llll}
1 & e^{j 2 \pi \theta^{(k)} / P} & \cdots & e^{j 2 \pi(P-1) \theta^{(k)} / P}
\end{array}\right]
\end{aligned}
$$

where the superscript $(\cdot)^{\mathrm{T}}$ denotes transpose and $\theta^{(k)}$ is

$$
\theta^{(k)}=\frac{q+\xi^{(k)}}{Q}
$$

We further define $\theta^{(k)}$ as the effective $C F O$ of the $k$ th user. As will be shown later, the proposed algorithm estimates the effective CFO rather than the normalized CFO directly.

Effective CFO has one important property. Different users have distinct effective CFOs. From its definition, we can show that if one user occupies subchannel $\{q\}$, the range of its effective $\mathrm{CFO}$ is $((q-0.5) / Q),(q+0.5) / Q))$, since the range 
of $\xi^{(k)}$ is $(-0.5,0.5)$. Because different users occupy different subchannels, their effective $\mathrm{CFO}$ s fall in nonoverlapping ranges.

\section{B. Multiple-User Signal Structure}

From (8), at the uplink receiver, the remaining $N$ superposed signal samples of one OFDMA block after the removal of CP are given by

$$
\Upsilon(n)=\sum_{k=1}^{K} \sum_{p=0}^{P^{(k)}-1} H_{p}^{(k)} X_{p}^{(k)} e^{j(2 \pi / N)\left(p Q+q^{(k)}+\xi^{(k)}\right) n}
$$

where the $k$ th user is assigned to subchannel $\left\{q^{(k)}\right\}$. In (16), we assume signals from different users are synchronized in time at the uplink receiver. The issue of time offsets among different users will be discussed in Section V-C.

$\{\Upsilon(n)\}_{n=0}^{N-1}$ can also be arranged into a $R \times P$ matrix, $\mathbf{A}$, in the same fashion as (13). From (14) and (16), the following relationship holds:

$$
\mathbf{A}=\sum_{k=1}^{K} \mathbf{A}^{(k)}=\mathbf{V S}=\mathbf{V}\{\mathbf{U} \odot(\mathbf{B W})\}
$$

where $\mathbf{S}=\mathbf{U} \odot(\mathbf{B W}) . \mathbf{W}$ is the same as in (14)

$$
\mathbf{U}=\left[\begin{array}{c}
\boldsymbol{u}^{(1)} \\
\boldsymbol{u}^{(2)} \\
\vdots \\
\boldsymbol{u}^{(K)}
\end{array}\right]_{K \times P} \quad \text { and } \quad \mathbf{B}=\left[\begin{array}{c}
\boldsymbol{b}^{(1)} \\
\boldsymbol{b}^{(2)} \\
\vdots \\
\boldsymbol{b}^{(K)}
\end{array}\right]_{K \times P} .
$$

$\mathbf{V}=\left[\boldsymbol{v}^{(1)}, \boldsymbol{v}^{(2)}, \ldots, \boldsymbol{v}^{(K)}\right]$ is a Vandermonde matrix with the following format:

$$
\left[\begin{array}{cccc}
1 & 1 & \cdots & 1 \\
e^{j 2 \pi \theta^{(1)}} & e^{j 2 \pi \theta^{(2)}} & \cdots & e^{j 2 \pi \theta^{(K)}} \\
\vdots & \vdots & \ddots & \vdots \\
e^{j 2 \pi(R-1) \theta^{(1)}} & e^{j 2 \pi(R-1) \theta^{(2)}} & \cdots & e^{j 2 \pi(R-1) \theta^{(K)}}
\end{array}\right]_{R \times K} .
$$

Finally, $\mathbf{S}$ is a $K \times P$ matrix, and its $k$ th row is $\left[\Upsilon^{(k)}(0), \Upsilon^{(k)}(1), \ldots, \Upsilon^{(k)}(P-1)\right]$

\section{CFO ESTIMATION AlgORITHM}

In this section, we estimate the CFOs of all involved users from the received signal samples of one OFDMA block based on (17). There are two requirements in determining the $\mathrm{CFO}$ for each user.

- Find $K$ estimates $\Delta \hat{f}^{(k)}, k=1,2, \ldots, K$, for $\Delta f^{(k)}$

- Match each $\Delta \hat{f}^{(k)}$ with the user it belongs to.

Due to the property of effective CFO, if subchannel $\{q\}$ is occupied by one user, there will be one and only one effective CFO which falls into the range of $((q-0.5) / Q),(q+0.5) / Q))$. It is thus a simple mapping to match the estimated effective CFOs with their corresponding users. We assume that the uplink receiver knows the number of users $K$, and how the subchannels are distributed among all users. Based on (15), the estimate of the CFO between the uplink receiver and the user assigned to subchannel $\{q\}$ is derived by

$$
\Delta \hat{f}^{(k)}=\hat{\xi}^{(k)} \Delta f=\left(Q \hat{\theta}^{(k)}-q\right) \Delta f
$$

where $\Delta \hat{f}^{(k)}, \hat{\xi}^{(k)}$, and $\hat{\theta}^{(k)}$ are the estimates of $\Delta f^{(k)}, \xi^{(k)}$, and $\theta^{(k)}$, respectively. In the following, we propose a structurebased blind algorithm to estimate the effective CFOs of all $K$ involved users simultaneously.

\section{A. A Structure-Based Estimation Algorithm}

A matrix form representation of an OFDMA block observed in noise is given by

$$
\mathbf{Y}=\mathbf{A}+\mathbf{Z}=\mathbf{V S}+\mathbf{Z}
$$

where $\mathbf{Z}$ is a $R \times P$ additive white Gaussian noise (AWGN) matrix. Each element of $\mathbf{Z}$ is a Gaussian random variable with zero mean and variance $\sigma^{2}$.

Let $\boldsymbol{y}_{l}, s_{l}$, and $z_{l}$ denote the $l$ th column of $\mathbf{Y}, \mathbf{S}$, and $\mathbf{Z}, l=$ $1,2, \ldots, P$. We have $\boldsymbol{y}_{l}=\mathbf{V} \boldsymbol{s}_{l}+z_{l}$. The covariance matrix of $\boldsymbol{y}_{l}$ is given by

$$
\boldsymbol{\Psi}=\mathrm{E}\left[\boldsymbol{y}_{l} \boldsymbol{y}_{l}^{\mathcal{H}}\right]=\mathbf{V} \boldsymbol{\Phi} \mathbf{V}^{\mathcal{H}}+\sigma^{2} \mathbf{I}
$$

where $(\cdot)^{\mathcal{H}}$ represents the Hermitian operation, and $\mathrm{E}[\cdot]$ means the expectation value. In (21), $\boldsymbol{\Phi}=\mathrm{E}\left[\boldsymbol{s}_{l} \boldsymbol{s}_{l}^{\mathcal{H}}\right]$ is the covariance matrix of $s_{l}$, and $\mathbf{I}$ is a $R \times R$ identity matrix.

From the definition, $\boldsymbol{\Psi}$ is a Hermitian matrix, so its eigenvalues are all positive real numbers [22]. Let $\lambda_{1} \geq \lambda_{2} \geq \cdots \geq$ $\lambda_{R}$ denote the $R$ eigenvalues of $\boldsymbol{\Psi}$ in descending order. Let $K<R$, we thus have $\lambda_{K+1}=\lambda_{K+2}=\cdots=\lambda_{R}=\sigma^{2}$ (see [20, App., R5]). Singular value decomposition (SVD) of $\boldsymbol{\Psi}$ is

$$
\Psi=\left[\begin{array}{ll}
U_{s} & \boldsymbol{U}_{z}
\end{array}\right]\left[\begin{array}{cc}
\boldsymbol{\Sigma}_{s} & \mathbf{0} \\
\mathbf{0} & \boldsymbol{\Sigma}_{z}
\end{array}\right]\left[\begin{array}{c}
U_{s}^{\mathcal{H}} \\
U_{z}^{\mathcal{H}}
\end{array}\right]
$$

where $\boldsymbol{\Sigma}_{s}=\operatorname{Diag}\left[\lambda_{1}, \ldots, \lambda_{K}\right]$ and $\boldsymbol{\Sigma}_{z}=\operatorname{Diag}\left[\lambda_{K+1}, \ldots\right.$, $\left.\lambda_{R}\right]$. $\boldsymbol{U}_{s}$ is a $R \times K$ matrix composed of $K$ eigenvectors corresponding to $\lambda_{1}, \lambda_{2}, \ldots, \lambda_{K}$, and $\boldsymbol{U}_{z}$ is a $R \times(R-K)$ matrix composed of $R-K$ eigenvectors corresponding to $\lambda_{K+1}, \ldots, \lambda_{R}$.

Since $\boldsymbol{U}_{s}$ and $\boldsymbol{U}_{z}$ are both unitary matrices, $\boldsymbol{U}_{s} \boldsymbol{U}_{s}^{\mathcal{H}}=$ $\boldsymbol{U}_{z} \boldsymbol{U}_{z}^{\mathcal{H}}=\mathbf{I}$. Let $\mathcal{S}$ denote the subspace spanned by $\boldsymbol{U}_{s}\left(\mathcal{S}=\operatorname{span}\left(\boldsymbol{U}_{s}\right)\right)$ and $\mathcal{Z}$ denote the subspace spanned by $\boldsymbol{U}_{z}\left(\mathcal{Z}=\operatorname{span}\left(\boldsymbol{U}_{z}\right)\right) . \mathcal{S}$ is the signal subspace of $\boldsymbol{\Psi}$, and $\mathcal{Z}$ is the noise subspace. $\mathcal{S}$ and $\mathcal{Z}$ are orthogonal to each other, and $\boldsymbol{U}_{s} \boldsymbol{U}_{z}^{\mathcal{H}}=\mathbf{0}$. From (22), we have

$$
\boldsymbol{\Psi}=\boldsymbol{U}_{s} \boldsymbol{\Sigma}_{s} \boldsymbol{U}_{s}^{\mathcal{H}}+\sigma^{2} \boldsymbol{U}_{z} \boldsymbol{U}_{z}^{\mathcal{H}} .
$$

Multiplying (21) and (23) by $\boldsymbol{U}_{z}$, respectively

$$
\begin{aligned}
& \boldsymbol{\Psi} \boldsymbol{U}_{z}=\mathbf{V} \boldsymbol{\Phi} \mathbf{V}^{\mathcal{H}} \boldsymbol{U}_{z}+\sigma^{2} \boldsymbol{U}_{z} \\
& \boldsymbol{\Psi} \boldsymbol{U}_{z}=\sigma^{2} \boldsymbol{U}_{z}
\end{aligned}
$$

we find $\mathbf{V} \boldsymbol{\Phi} \mathbf{V}^{\mathcal{H}} \boldsymbol{U}_{z}=\mathbf{0}$. Vandermonde matrix $\mathbf{V}$ is full rank [20]. We assume users are independent and signals are generated independently, so that covariance matrix $\boldsymbol{\Phi}$ is also full rank. Hence, $\mathbf{V} \boldsymbol{\Phi}$ is full rank, and $\mathbf{V}^{\mathcal{H}} \boldsymbol{U}_{z}=\mathbf{0}$. For each column 
of $\mathbf{V}, \mathbf{v}_{k}$, where $k=1, \ldots, K$, we have $\boldsymbol{U}_{z}^{\mathcal{H}} \mathbf{v}_{k}=0$, and thus $\left\{\theta^{(k)}\right\}_{k=1}^{K}$ correspond to the largest $K$ local maximum of

$$
\frac{1}{\left\|\mathbf{a}^{\mathcal{H}}(\theta) \boldsymbol{U}_{z} \boldsymbol{U}_{z}^{\mathcal{H}} \mathbf{a}(\theta)\right\|^{2}}
$$

where $\mathbf{a}(\theta)=\left[1, e^{j 2 \pi \theta}, \ldots, e^{j 2 \pi(R-1) \theta}\right]^{T}$.

In (20), $\mathbf{Y}$ is a $R \times P$ matrix, where $P$ is the number of subcarriers in one subchannel. In an OFDMA system with 2048 subcarriers, if there are 16 subchannels, $P=128$; if there are 32 subchannels, $P=64$. In Section IV-B, diversity schemes will be introduced where the number of columns in $\mathbf{Y}$ is the multiple of $P$. With a large number of columns in $\mathbf{Y}$, the covariance matrix $\boldsymbol{\Psi}$ can be estimated by

$$
\widehat{\mathbf{\Psi}}=\frac{1}{P} \mathbf{Y} \mathbf{Y}^{\mathcal{H}}=\frac{1}{P} \sum_{l=1}^{P} \boldsymbol{y}_{l} \boldsymbol{y}_{l}^{\mathcal{H}} .
$$

And the estimation of $\boldsymbol{U}_{s}$ and $\boldsymbol{U}_{z}, \hat{\boldsymbol{U}}_{s}$, and $\hat{\boldsymbol{U}}_{z}$ can be derived by introducing SVD to $\hat{\Psi}$.

In summary, the CFOs of all $K$ users are estimated using the signal samples of one OFDMA block as shown in the following. Step 1) Formulate the received signal samples into matrix form $\mathbf{Y}$.

Step 2) Introduce SVD to $\hat{\boldsymbol{\Psi}}=\mathbf{Y} \mathbf{Y}^{\mathcal{H}} / P$ to find $\hat{\boldsymbol{U}}_{z}$.

Step 3) Find the largest $K$ peaks of (25) to estimate $\left\{\theta^{(k)}\right\}_{k=1}^{K}$.

Step 4) Calculate $\Delta f^{(k)}$ using (19).

Neither the channel knowledge nor the transmitted training symbols are required in the proposed structure-based algorithm. In this sense, the proposed algorithm is a blind method for the estimation problem of concern.

From (25), the rank of the noise subspace $\mathcal{Z}$ is required to be at least one. In other words, we assume $R>K$. There are two methods for satisfying this requirement. In the first method, the maximum number of supported users in one OFDMA block $K_{\max }$, is set to $K_{\max }=Q-1<Q$. For an OFDMA system with $Q$ subchannels, $R=Q$. Hence, $R>K$ is satisfied. In the second method, we let $K_{\max }=Q$ and extend the length of CP to $N_{\text {cp }}+\mu P$, where $\mu$ is a positive integer. The first $N_{\mathrm{cp}}$ samples are the original $\mathrm{CP}$ and are used to accommodate both channel delay spreads and timing offsets among multiple users [23]. The following $\mu P$ samples are not contaminated by IBI from the previous OFDMA block and used by the structurebased algorithm with the remaining $N$ samples for estimation, such that $R=(N+\mu P) / P=Q+\mu>K_{\max }$.

In terms of the subspace-based analysis, the proposed algorithm is similar to the methods used in multiple signal classification (MUSIC) [24] and estimation of signal parameters by rotational invariance techniques (ESPRIT) [25]. In [19], MUSIC and ESPRIT were used in a statistics-based algorithm to estimate the CFOs in the uplink of OFDMA, in which the number of unknown parameters is equal to the number of subcarriers in the system. Therefore, its application is confined to OFDMA with a limited number of subcarriers. Furthermore, since one user occupies a set of subcarriers, the statistics-based algorithm will give an estimate of its CFO per subcarrier. Combining of the estimates from the subcarriers is inevitable for each user to give the final estimation, and the optimal combining method requires additional knowledge, such as channel information or modulation symbols [26].

Unlike the statistics-based algorithm, the structure-based algorithm proposed in this paper takes advantage of the inner algebraic structure of interleaved OFDMA signals in one OFDMA block, i.e., the periodic property of each user's signal, such that it only estimates one CFO for each user, regardless of the number of subcarriers one user occupies. It is computationally efficient for OFDMA systems with large subcarrier size. Secondly, it is applicable to the environment where system parameters or channel conditions are changing from block to block, in which the statistics-based algorithm is not feasible. In Section V, we will show the accuracy of the structure-based algorithm is also better than that of the statistics-based algorithm in [19].

\section{B. Estimation With Diversity}

In the above, only one OFDMA block is used in the structure-based algorithm. Under moderate or high SNR, the signal samples of one OFDMA block are sufficient for accurate CFO estimation, as will be shown later in simulation results. However, due to the limited samples, the covariance matrix of the sampled noise is not ideally diagonal. Under low SNR conditions, this will degrade the performance of the proposed estimation algorithm.

Diversity schemes can be applied to increase the amount of signal samples, as well as the amount of noise samples, for estimation purposes. If the diversity scheme is selected such that all noise samples are uncorrelated, the effect of the noise is closer to that of ideal white noise, and the performance of the structurebased estimation algorithm is thus improved. Estimation using multiple OFDMA blocks [27] and multiple receive antennas [28] are two feasible diversity schemes. Oversampling is not appropriate, since it samples the received signals using a rate higher than the Nyquist rate and introduces correlation among noise samples. This leads to colored noise. In fact, subspace-based methods perform poorly when the noise is not white [20].

Let $\{m\}_{m=1}^{M}$ denote the index of received OFDMA blocks. In the multiple OFDMA block-diversity scheme, $\left\{\mathbf{Y}_{m}\right\}_{m=1}^{M}$ are the consecutive OFDMA blocks received by the uplink receiver. In the multiple receive-antenna diversity scheme, $\mathbf{Y}_{m}$ denotes the signal matrix of the OFDMA block received from the $m$ th antenna. We have

$$
\mathbf{Y}_{m}=\mathbf{V S}_{m}+\mathbf{Z}_{m}
$$

where $\mathbf{S}_{m}$ is the same as the $\mathbf{S}$ defined in (17), except that the subscript $(\cdot)_{m}$ is the index of the OFDMA block, and $\mathbf{Z}_{m}$ is the noise matrix sampled in the $m$ th OFDMA block. Define $\tilde{\mathbf{Y}}=\left[\begin{array}{llll}\mathbf{Y}_{1} & \mathbf{Y}_{2} & \cdots & \mathbf{Y}_{M}\end{array}\right]$. We have

$$
\tilde{\mathbf{Y}}=\mathbf{V} \tilde{\mathbf{S}}+\tilde{\mathbf{Z}}
$$

where $\tilde{\mathbf{S}}=\left[\begin{array}{llll}\mathbf{S}_{1} & \mathbf{S}_{2} & \cdots & \mathbf{S}_{M}\end{array}\right]$ and $\tilde{\mathbf{Z}}=\left[\begin{array}{llll}\mathbf{Z}_{1} & \mathbf{Z}_{2} & \cdots & \mathbf{Z}_{M}\end{array}\right]$.

Let $\tilde{\boldsymbol{\Psi}}=\tilde{\mathbf{Y}} \tilde{\mathbf{Y}}^{\mathcal{H}} / P$. Applying the proposed algorithm to $\tilde{\boldsymbol{\Psi}}$, we will show the improvement of the estimation performance through simulation in Section V. 


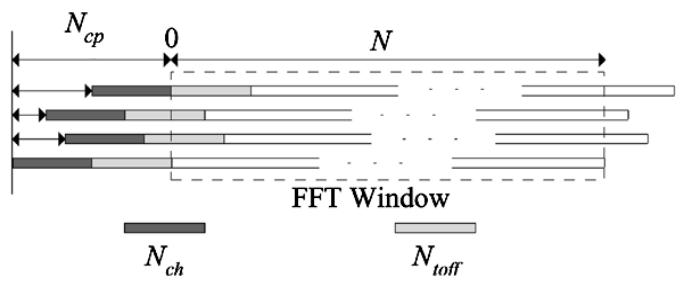

Fig. 2. $\mathrm{CP}$ and uplink receiver FFT window.

\section{Estimation in the Presence of Time Offset}

In the above, we assume that all users are time synchronized. In a practical wireless environment, OFDMA blocks from distinct users are offset with each other when they arrive at the uplink receiver, due to their different propagation delays. The time offsets among users will introduce IBI and degrade the performance of OFDMA.

A common way to compensate for the time offsets among users is to select the length of the $\mathrm{CP}$ to accommodate both the maximum channel delay spread and the maximum time offset [23]. This is shown in Fig. 2. The CP is composed of two parts, as $N_{\text {cp }}=N_{\text {ch }}+N_{\text {toff }}$, where $N_{\mathrm{ch}}$ is the portion of the CP for accommodating channel delay spreads, while the additional $N_{\text {toff }}$ samples are intended for accommodating different time offsets among users. The vertical line on the left of Fig. 2 is the starting point of an OFDMA block at the uplink receiver. OFDMA blocks from distinct users arrive at the receiver with different delays. If $N_{\text {toff }}$ is selected to be the maximum possible delay among all users, and the uplink receiver FFT demodulation window starts from the zero position as in Fig. 2, the $N$ superposed signal samples that in the window are immune to IBI. An approach in which $N_{\text {toff }}$ is shorter than the maximum time delay was introduced in [29] to increase the efficiency and minimize the residual interference.

Based on the aforementioned technique, we assume that the $N$ superposed signal samples that the uplink receiver used for FFT demodulation, $\{\Upsilon(n)\}_{n=0}^{N-1}$, are not contaminated by IBI. Let $d^{(k)}$ denote the delay time between the $k$ th user and the uplink receiver, in terms of the number of signal samples and $\left\{\Upsilon^{\prime(k)}(n)\right\}_{n=0}^{N-1}$ denote the $N$ signal samples from the $k$ th user that contribute to $\{\Upsilon(n)\}_{n=0}^{N-1}$. We have

$$
\begin{aligned}
\Upsilon^{\prime(k)}(n) & =\sum_{p=0}^{P-1} H_{p}^{(k)} X_{p}^{(k)} e^{j \frac{2 \pi}{N}(p Q+q)\left(n-d^{(k)}\right)} e^{j \frac{2 \pi}{N} \xi^{(k)} n} \\
& =e^{j(2 \pi / N) \xi^{(k)} d^{(k)}} \Upsilon^{(k)}\left(n-d^{(k)}\right)
\end{aligned}
$$

where $n=0,1, \ldots, N-1$ and $\Upsilon^{(k)}(n)$ is defined in (10). Similar to (11) and (12), through simple derivations we have $\Upsilon^{\prime(k)}(n+\nu P)=e^{j 2 \pi \nu \theta^{(k)}} \Upsilon^{\prime(k)}(n)$, which means the periodic structure still exists in $\left\{\Upsilon^{\prime(k)}(n)\right\}_{n=0}^{N-1}$. Therefore, the proposed structure-based algorithm is applicable when signals from different users arrive at the uplink receiver asynchronously.

\section{Simulation Results}

In this section, we provide the simulation results of the proposed structure-based estimation algorithm.

\section{A. System Scenario and Channel Model}

The transmission parameters of the OFDMA system in our simulation are selected from IEEE 802.16a [6]. The uplink bandwidth (BW) is $20 \mathrm{MHz}$, and the FFT size is $N=2048$. The sampling frequency $F_{s}$ is determined by $F_{s}=\mathrm{BW} \cdot 8 / 7$, hence, the subcarrier spacing $\Delta f$ is $11.16 \mathrm{kHz}$ and $T=89.6 \mu \mathrm{s}$. Within the 2048 subcarriers, there are 176 virtual subcarriers on the left-side band and 175 on the right. The length of a period within one OFDMA block is determined by the configuration of subchannels. If the 2048 subcarriers are divided into 16 subchannels, the length of a period is $5.6 \mu \mathrm{s}$; for 32 subchannels, it is $2.8 \mu \mathrm{s}$. The CP is composed of 256 samples, where the first 128 samples are the original $\mathrm{CP}$ portion for mitigating the channel delay spreads and the time offsets among users. The remaining 128 samples are used by the proposed CFO estimation algorithm, such that $R>K_{\max }$ and $K_{\max }=Q$ in the following simulations.

The time-varying multipath channel in the simulation is described in (4). The probability distribution for the delay lags is

$$
f\left(\tau_{i}\right)= \begin{cases}1 / D, & \text { if } \tau_{i} \in[0, D] \\ 0, & \text { otherwise }\end{cases}
$$

where $D$ is the maximum path delay in terms of sample intervals and $\tau_{i}$ can be a noninteger value. The power-delay profile of $\alpha_{i}$ is [30]

$$
\eta_{\tau}=C e^{-\tau / \tau_{\mathrm{rms}}}
$$

where $C$ is the average power of the first path.

In the simulations, the maximum number of fading paths for each user is six. In each Monte Carlo test, $L^{(k)}$ is a randomly generated integer between $[1,6]$ for each user. $C$ is set to be 1 and $\tau_{\text {rms }}$ is $1.4 \mu \mathrm{s}$. The simulated channels also included the effect of the pulse-shaping filters at both the transmitter and the receiver, which are square root-raised cosine filters. The combination of the maximum channel length and the maximum time offset among users is no longer than 128 sample intervals.

\section{B. Algorithm Performance}

The performance of the effective CFO estimation determines the accuracy of the CFO estimation. In Fig. 3, we show the mean square error (MSE) performance of the effective CFO estimation in three different cases, and compare them with the corresponding Cramér-Rao bounds (CRB). The multiuser CRB of the estimation algorithm is given in [31, eq. (4.6)]. Each user's CRB depends on its CFO and subchannel allocation, the total number of users, and the CFO of its neighboring users. For illustration purposes, we assume the systems are fully loaded $(K=Q)$ in all three cases shown in Fig. 3, and each user has the same amount of CFO, which is set as $\xi=0.2$. Thus, the $\mathrm{CRB}$ of each user is the same. The MSE of the effective CFO estimation is determined by

$$
\frac{1}{K \Pi} \sum_{\rho=1}^{\Pi} \sum_{k=1}^{K}\left[\hat{\theta}_{\rho}^{(k)}-\theta^{(k)}\right]^{2}
$$

where $\hat{\theta}_{\rho}^{(k)}$ is the estimation of effective CFO of the $k$ th user at the $\rho$ th Monte Carlo simulation, and $\Pi$ is the total number 


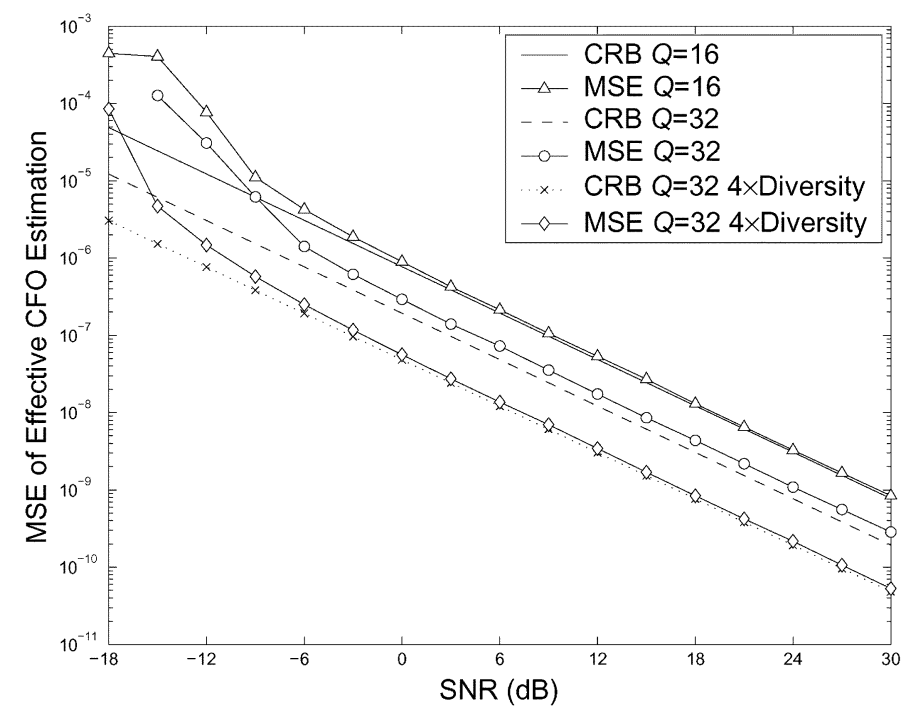

Fig. 3. MSE performance of effective CFO estimation compared with the CRB.

of Monte Carlo tests. From the figure, when $Q=16$, the estimation is optimal even without diversity schemes; while for $Q=32$, the four-times diversity case outperforms the no-diversity case, since its MSE curve is much closer to the CRB. For all three cases, the MSE curves of the effective CFO estimation approach their best performance for SNR greater than $-6 \mathrm{~dB}$.

To quantify the performance of the CFO estimation, the normalized root mean square error (RMSE) of the estimates is used, and it is defined as

$$
\text { Normalized RMSE }=\sqrt{\frac{1}{K \Pi} \sum_{\rho=1}^{\Pi} \sum_{k=1}^{K}\left[\hat{\xi}_{\rho}^{(k)}-\xi_{\rho}^{(k)}\right]^{2}}
$$

where $K$ is the number of users, $\Pi$ is the total number of Monte Carlo tests, and $\hat{\xi}_{\rho}^{(k)}$ is the estimate of $\xi_{\rho}^{(k)}$. The subscript $(\cdot)_{\rho}$ denotes the index of the Monte Carlo test. In each Monte Carlo test, we randomly generate a CFO and a multipath channel for each user. The normalized RMSE is computed after averaging over all participating users for 400 independent Monte Carlo tests. All user data symbols were independent quaternary (Q)PSK symbols. The SNR of the $k$ th user is defined as $\mathrm{SNR}^{(k)}=\mathrm{E}\left[\left|\Upsilon^{(k)}(n)\right|^{2}\right] / \sigma^{2}$

Example 1: In this example, we applied the proposed structure-based algorithm using the signal samples of one OFDMA block. All $\mathrm{SNR}^{(k)}, k=1,2, \ldots, K$ are assumed to be same at the uplink receiver, and each user only occupies one subchannel in one OFDMA block. First, we divided the 2048 subcarriers into 16 subchannels and calculated the normalized RMSE of the estimation algorithm for $K=4,8,12$, respectively. The results are presented in Fig. 4(a). In Fig. 4(b), the 2048 subcarriers were divided into 32 subchannels, and the normalized RMSE is plotted for $K=16,20,24$, respectively.

We observe that the normalized RMSE of the proposed algorithm increases when the number of users in one OFDMA block increases. If the OFDMA system is operating in moderate or high SNR (10 dB or higher), the normalized RMSE of the proposed algorithm is less than $1 \%$ of the subcarrier spacing. If the uplink receiver SNR is low, such as $3 \mathrm{~dB}$, the normalized RMSE is only around $2 \%$ of the subcarrier spacing. The sensitivity of

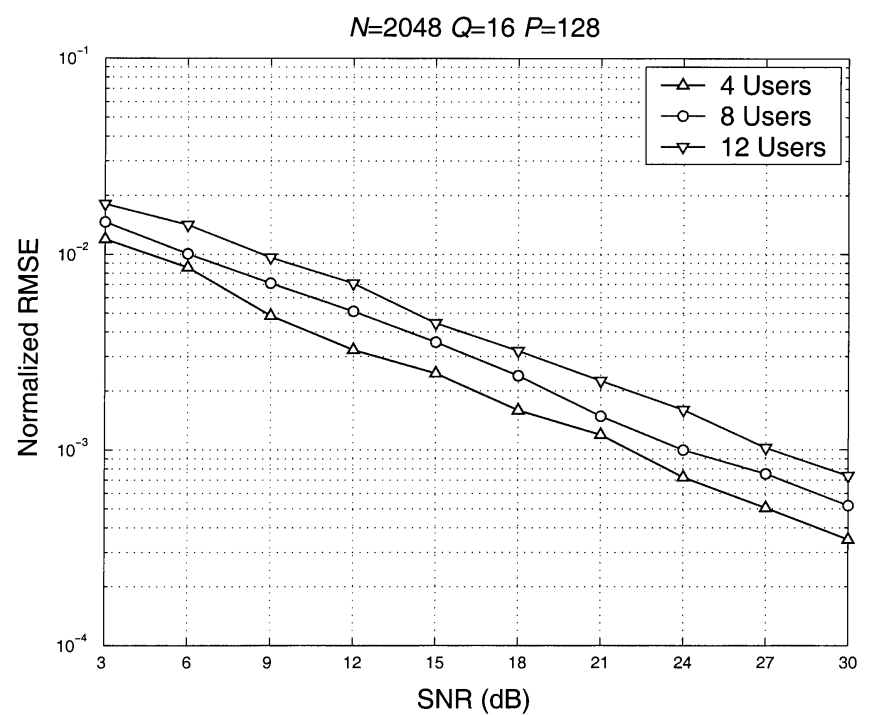

(a)

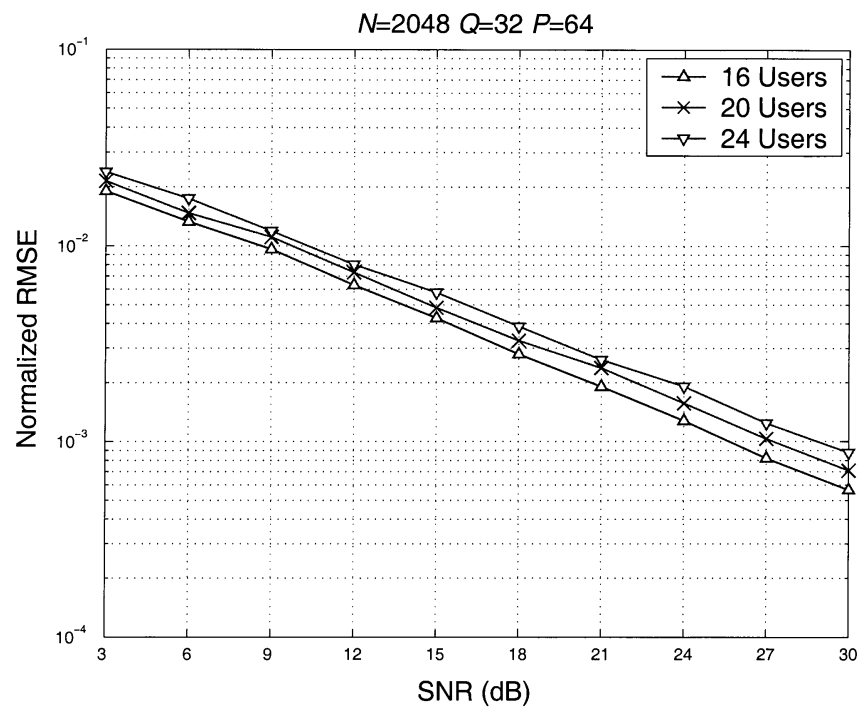

(b)

Fig. 4. Normalized RMSE vs. SNR when a different number of users are present in one OFDMA block. (a) 16 subchannels, each subchannel has 128 subcarriers. (b) 32 subchannels, each subchannel has 64 subcarriers.

the OFDMA system to CFO depends on SNR. The system performance degradation is more serious under high SNR than low SNR when the same amount of normalized CFO is presented [8], [14]. In fact, when the receiver SNR of the OFDMA system is lower than $10 \mathrm{~dB}$, the system degradation caused by $\mathrm{CFO}$ smaller than 5\% is less than $1 \mathrm{~dB}$ in output SNR [8].

The estimation error for a single user, $\epsilon^{(k)}=\hat{\xi}^{(k)}-\xi^{(k)}$, in each simulation test is also recorded, and its distribution in different cases are presented in Fig. 5. It is possible that, in one simulation test, the estimates for a few users may have large errors, such as $10 \%$ of the subcarrier spacing, while the estimation errors for the other users in the same OFDMA block are less than $1 \%$ of the subcarrier spacing. From Fig. 5, we observed that when the receiver SNR increases, the distribution of estimation error $\epsilon$ is more centralized.

Particularly, the estimated effective CFO of a user at subchannel $\{q\}$ may exceed the range of $((q-0.5) / Q),(q+$ $0.5) / Q)]$ under low SNR. This kind of estimation error is 

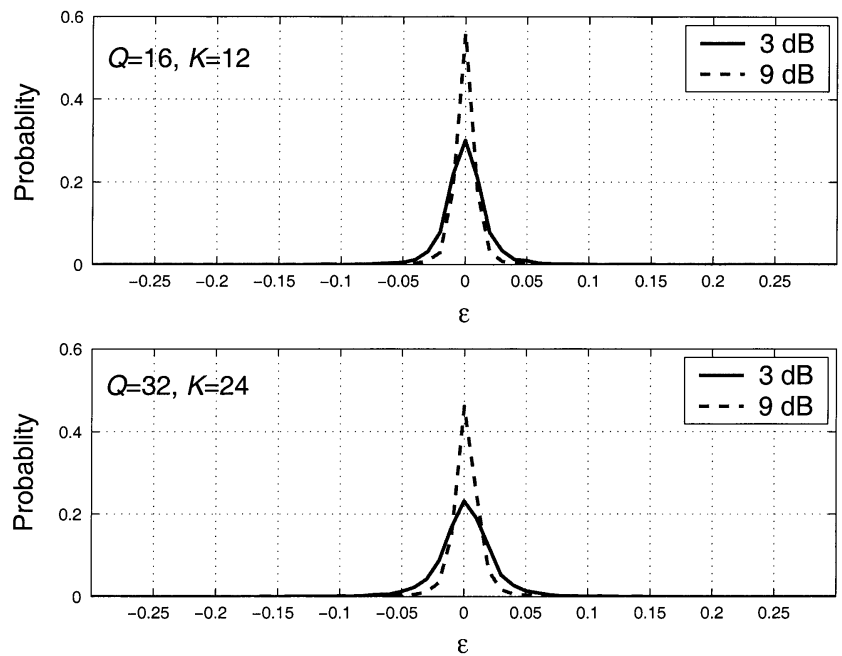

Fig. 5. Distribution of $\epsilon^{(k)}=\hat{\xi}^{(k)}-\xi^{(k)}$. Top: 16 subchannels and 12 users. Bottom: 32 subchannels and 24 users.

TABLE I

MISMATCH PROBABILITY

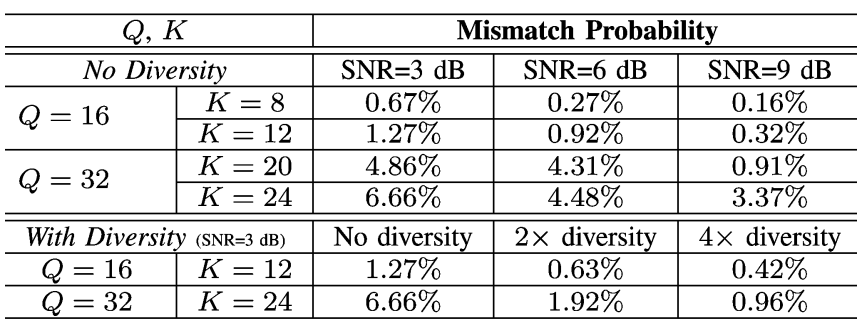

defined as mismatch. In Table I, we collected the probability of mismatch observed from the simulation. Notice that when 24 users are simultaneously transmitting signals in an OFDMA system with 32 subchannels, the mismatch probability is relatively high if the SNR is low. This can be alleviated by incorporating diversity schemes, as shown below.

Example 2: In this example, we investigate the performance of the proposed algorithm when diversity schemes are deployed at the uplink receiver. Multiple receive antennas are applied. The estimations are carried out using two receive antennas (4096 signal samples, diversity order 2) and four receiver antennas (8192 signal samples, diversity order 4). The normalized RMSE are plotted in Fig. 6(a) for 12 users in a 16-subchannels setup and in Fig. 6(b) for 24 users in a 32-subchannels setup. The normalized RMSE without diversity scheme (2048 signal samples) are also plotted in each case for comparison.

As expected, the mismatch probability of the proposed algorithm decreases when diversity schemes are adopted and the results are also listed in Table I. Note that in the case of 32 subchannels and 24 users, the mismatch probability decreases to $0.96 \%$ when four-branch diversity is adopted under $3-\mathrm{dB}$ SNR. This indicates that the proposed algorithm is applicable for low SNR.

Example 3: In Fig. 7, we show the change of the normalized RMSE performance of the CFO estimation according to the variation of the system load. The system load is defined here as the ratio of the number of concurrent active users in one OFDMA block versus the maximum number of users supported by one block. In this simulation, it is given by $\eta=K / K_{\max }$

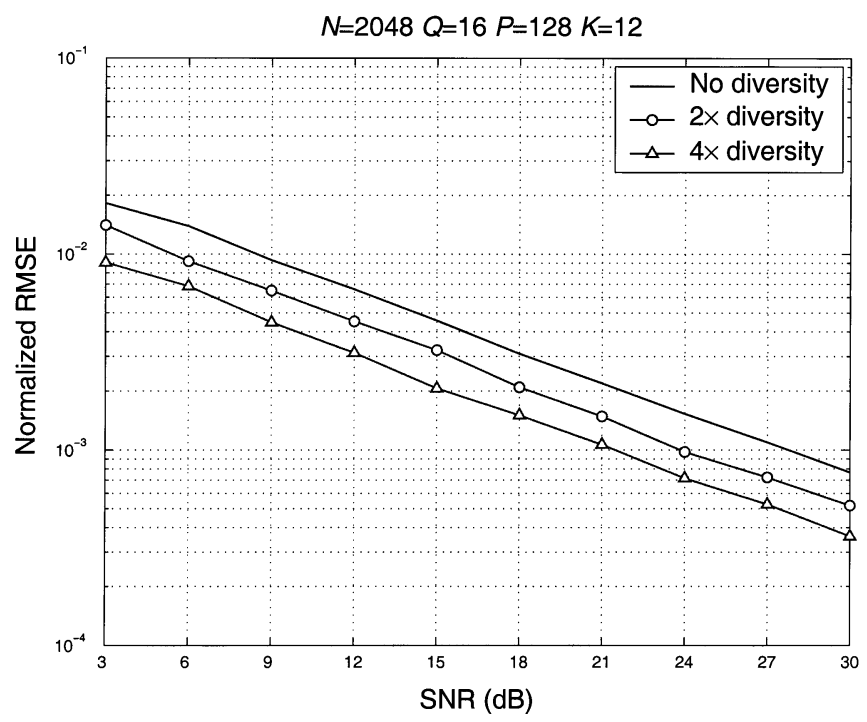

(a)

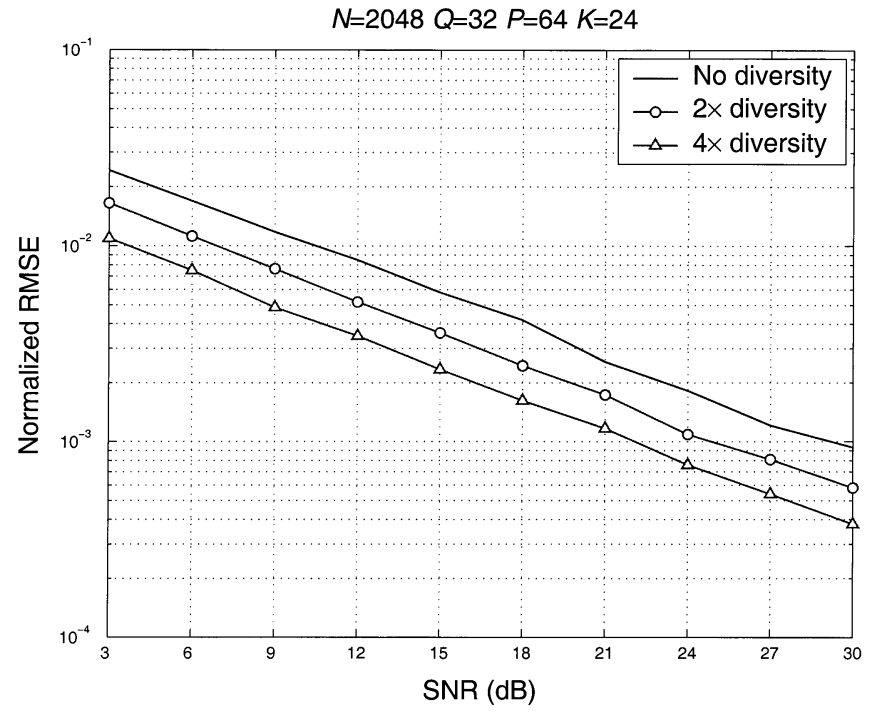

(b)

Fig. 6. Normalized RMSE versus SNR when diversity schemes are adopted at the uplink receiver. No diversity, $2 \times$ diversity, and $4 \times$ diversity are compared. (a) 16 subchannels and 12 users. (b) 32 subchannels and 24 users.

and expressed in percentage values. From Fig. 7, the normalized RMSE is a monotonically increasing function of $\eta$, hence, the lower the number of users in one OFDMA block, the better the performance. The slope of the curves illustrate how fast the performance is decreasing with the increasing of system load. It is observed from Fig. 7 that the normalized RMSE performance of the system with $Q=16$ declines faster than the system with $Q=32$.

Example 4: In this example, we illustrate the usefulness of the estimation algorithm in terms of the receiver BER performance improvement. Based on the estimated CFO, we adopted the minimum mean-square error (MMSE)-synchronization algorithm proposed in [18] to restore the orthogonality among all users. The simulation results are given in Fig. 8. In order to show the performance improvement solely resulting from the CFO estimation and the frequency synchronization, we select the uncoded BER as the measurement. The channel knowledge is assumed to be perfectly known. For comparison 


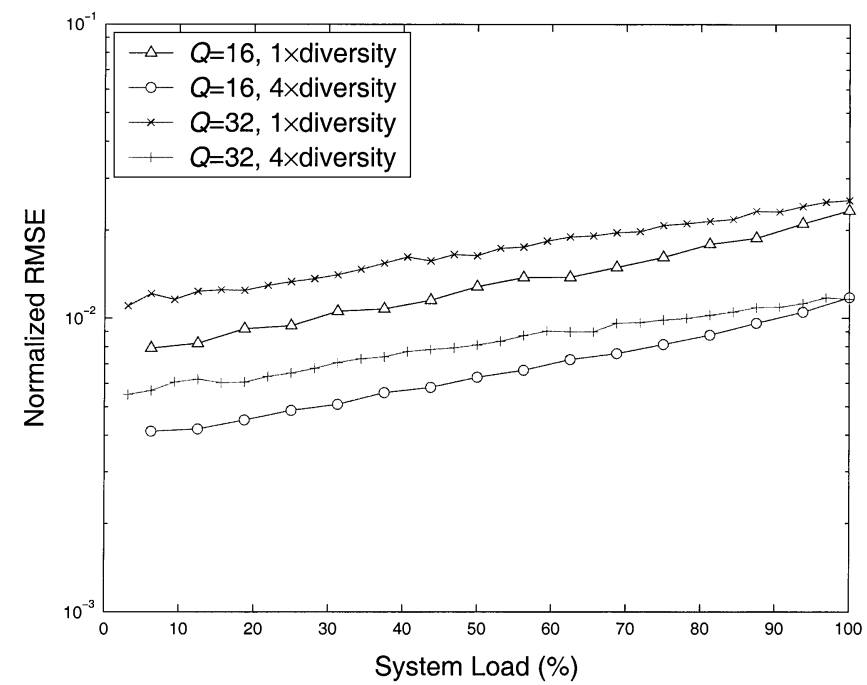

Fig. 7. Normalized RMSE performance versus the system load (SNR $=5$ $\mathrm{dB}$ ). The system load is defined as the ratio of the current active users in one OFDMA block and the maximum number of supported users in a block.

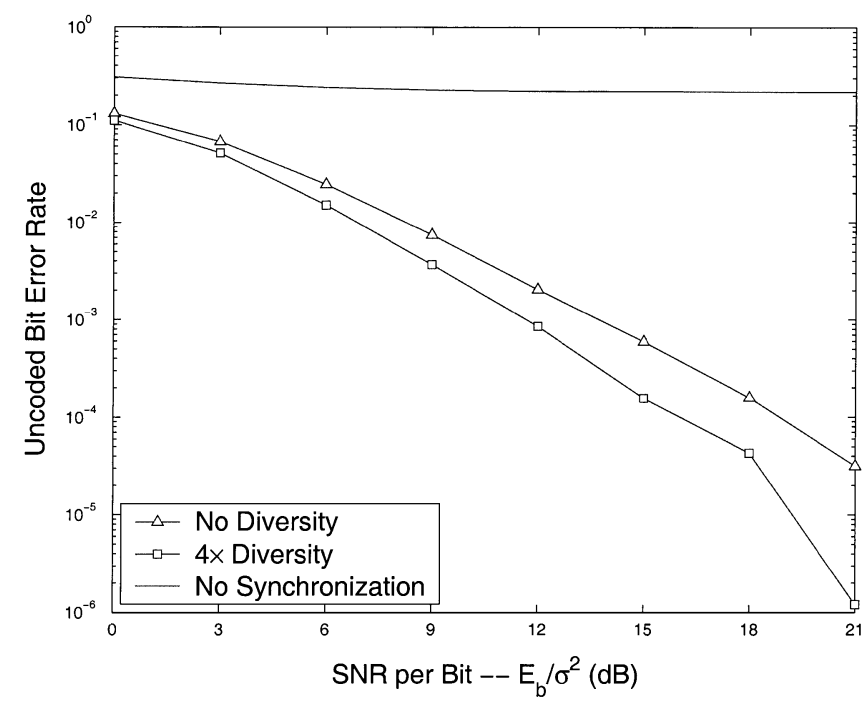

Fig. 8. Uncoded BER performance versus the per-bit SNR. $Q=32$ and $K=$ 24.

purposes, we also plotted the uncoded BER if there is no synchronization effort. It is shown that, without synchronization effort, the uncoded BER has an error floor bounded around 0.2 . With the MMSE-synchronization algorithm based on the estimation of CFO, the BER curves manifest a significant improvement. Applying diversity schemes to get more accurate estimates of CFO, the BER performance is superior to that without diversity schemes, as expected.

\section{Comparison}

In this part, we compare the performance of the proposed structure-based estimation algorithm with that of the statisticsbased algorithm proposed in [19] in an AWGN environment.

The simulated OFDMA system has 64 subcarriers and eight users. Each user occupies eight subcarriers in an interleaved fashion. CP is 24 , in which the first 16 samples are intended for eliminating the effect of channel delay spread. The last eight signal samples of $\mathrm{CP}$ and the remaining 64 signal samples of the

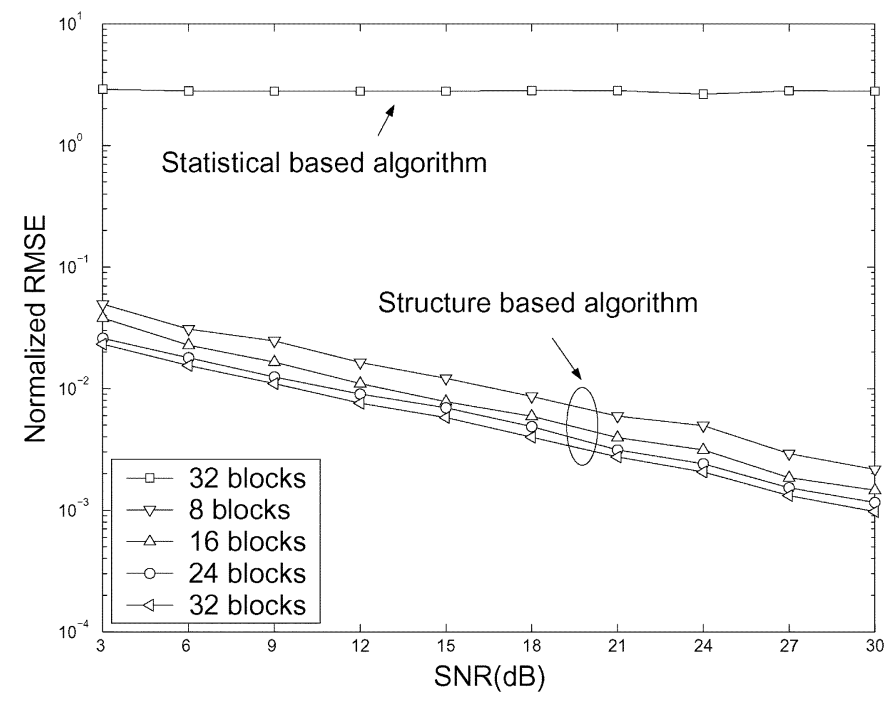

Fig. 9. Comparison between the proposed structure-based algorithm and the statistical-based algorithm in [19].

OFDMA compose a $9 \times 8$ signal matrix for the structure-based algorithm.

The statistics required by the algorithm in [19] are calculated over 32 consecutive OFDMA blocks including CP. We simulate the structure-based algorithm by using the first $8,16,24$, and all 32 OFDMA blocks, respectively. The resulting normalized RMSE is shown in Fig. 9.

Due to the presence of bias, the normalized RMSE of the statistics-based algorithm is around three, and more or less independent of SNR. Note that the simulation results presented in [19] is the MSE of the estimated CFO. For fair comparison, we presented the normalized RMSE for both algorithms, which is the square root of the MSE multiplied by the number of subcarriers $N$. From Fig. 9, the structure-based algorithm proposed in this paper outperforms the statistics-based algorithm.

\section{CONCLUSION}

A signal structure-based deterministic estimation algorithm is proposed in this paper for estimating the CFOs of all users simultaneously in the uplink of OFDMA systems with the interleaved subcarrier assignment. The inner algebraic structure of the signals at the uplink receiver is exploited for CFO estimation. The algorithm is computationally efficient in that it only requires signal samples of one OFDMA block. Simulation results indicate that it performs well, even under low SNR with diversity schemes. The algorithm presented here shows great potential for OFDMA systems using the interleaved subcarrier assignment in the uplink to reap diversity gains in frequency-selective fading channels.

\section{ACKNOWLEDGMENT}

The authors would like to thank the reviewers and the paper editor for their valuable comments and suggestions.

\section{REFERENCES}

[1] J. Bingham, "Multicarrier modulation for data transmission: An idea whose time has come," IEEE Commun. Mag., vol. 28, pp. 5-14, May 1990. 
[2] Digital Audio Broadcasting (DAB) to Mobile, Portable, and Fixed Receiver, ETSI Std. ETS 300 401, May 2001.

[3] Digital Video Broadcasting (DVB-T); Frame Structure, Channel Coding, and Modulation for Digital Terrestrial Television, ETSI Std. ETS 300 744, Dec. 2001.

[4] Supplement to IEEE Std. 802.11, Wireless LAN Media Access Control (MAC) and Physical Layer (PHY) Specifications: High-Speed Physical Layer in the 5 GHZ Band, IEEE Std. 802.11a, 2001.

[5] Broadband Radio Access Network (BRAN): HIPERLAN Type 2 Functional Specification Part 1-Physical Layer, ETSI Std. ETS/BRAN 030 003-1, June 1999.

[6] Air Interface for Fixed Broadband Wireless Access Systems-Amendment 2: Medium Access Control Modifications and Additional Physical Layer Specifications for 2-11 GHz, IEEE Std. 802.16a, 2003.

[7] T. Pollet, M. V. Bladel, and M. Moeneclaey, "BER sensitivity of OFDM systems to carrier frequency offset and Wiener phase noise," IEEE Trans. Commun., vol. 43, pp. 191-193, Feb.-Apr. 1995.

[8] A. M. Tonello, N. Laurenti, and S. Pupolin, "Analysis of the uplink of an asynchronous multi-user DMT OFDMA system impaired by time offsets, frequency offsets, and multi-path fading," in Proc. Vehicular Technology Conf.-Fall, vol. 3, Oct. 2000, pp. 1094-1099.

[9] J. Li, G. Liu, and G. B. Giannakis, "Carrier frequency offset estimation for OFDM-based WLANs," IEEE Signal Processing Lett., vol. 8, pp. 80-82, Mar. 2001.

[10] U. Tureli, H. Liu, and M. D. Zoltowski, "OFDM blind carrier offset estimation: ESPRIT," IEEE Trans. Commun., vol. 48, pp. 1459-1461, Sept. 2001.

[11] H. Liu and U. Tureli, "A high-efficiency carrier estimator for OFDM communication," IEEE Commun. Lett., vol. 2, pp. 104-106, Apr. 1998.

[12] T. M. Schmidl and D. C. Cox, "Blind synchronization for OFDM," Electron. Lett., vol. 33, no. 2, pp. 113-114, Feb. 1997.

[13] M. Luise and R. Reggiannini, "Carrier frequency acquisition and tracking for OFDM systems," IEEE Trans. Commun., vol. 44, pp. 1590-1598, Nov. 1996.

[14] P. H. Moose, "A technique for orthogonal frequency-division multiplexing frequency-offset correction," IEEE Trans. Commun., vol. 42, pp. 2908-2914, Oct. 1994.

[15] J. J. Beek, P. O. Börjesson, M. L. Boucheret, D. Landström, J. Arenas, P. Ödling, C. Östberg, M. Mahlqvist, and S. K. Wilson, "A time and frequency synchronization scheme for multiuser OFDM," IEEE J. Select. Areas Commun., vol. 17, pp. 1900-1914, Nov. 1999.

[16] J. Choi, C. Lee, H. W. Jung, and Y. H. Lee, "Carrier frequency offset compensation for uplink of OFDM-FDMA systems," IEEE Commun. Lett., vol. 4, pp. 414-416, Dec. 2000.

[17] S. Barbarossa, M. Pompili, and G. B. Giannakis, "Channel-independent synchronization of orthogonal frequency-division multiple access systems," IEEE J. Select. Areas Commun., vol. 20, pp. 474-486, Feb. 2002.

[18] Z. Cao, U. Tureli, and Y. D. Yao, "Analysis of two receiver schemes for interleaved OFDMA uplink," in Proc. 36th Asilomar Conf. Signals, Systems, Computers, Nov. 2002, pp. 1818-1821.

[19] H. Bölcskei, "Blind high-resolution uplink synchronization of OFDMbased multiple access schemes," in Proc. 2nd IEEE Workshop Signal Processing Advances in Wireless Communications, 1999, pp. 166-169.

[20] P. Stoica and R. Moses, Introduction to Spectral Analysis. Englewood Cliffs, NJ: Prentice-Hall, 1997.

[21] U. Tureli, D. Kivanc, and H. Liu, "Experimental and analytical studies on a high resolution OFDM carrier frequency offset estimator," IEEE Trans. Veh. Technol., vol. 50, pp. 629-643, Mar. 2001.

[22] J. M. Ortega, Matrix Theory. New York: Plenum, 1987.

[23] S. Kapoor, D. J. Marchok, and Y.-F. Huang, "Adaptive interference suppression in multiuser wireless OFDM systems using antenna arrays," IEEE Trans. Signal Processing, vol. 47, pp. 3381-3391, Dec. 1999.

[24] R. O. Schmidt, "Multiple emitter location and signal parameter estimation," in Proc. RADC Spectral Estimation Workshop, 1979, pp. $243-258$.

[25] R. Roy, A. Paulraj, and T. Kailath, "ESPRIT—A subspace rotation approach to estimation of parameters of cisoids in noise," IEEE Trans. Acoust., Speech, Signal Processing, vol. ASSP-34, pp. 1340-1342, Feb. 1986.

[26] H. Li, P. Stoica, and J. Li, "Computationally efficient parameter estimation for harmonic sinusoidal signals," Signal Processing, vol. 80, no. 11, pp. 1937-1944, 2000.

[27] B. Chen and H. Wang, "Maximum-likelihood estimation of OFDM carrier frequency offset," in Proc. IEEE Int. Conf. Communications, vol. 1, May 2002, pp. 49-53.

[28] U. Tureli, D. Kivanc, and H. Liu, "Multicarrier synchronization with diversity," in Proc. Vehicular Technology Conf., vol. 2, Oct. 2001, pp. $146-155$.

[29] S. Kaiser and W. A. Krzymien, "Performance effects of the uplink asynchronism in a spread spectrum multicarrier multiple access system," Eur. Trans. Commun., vol. 10, no. 4, pp. 399-406, July-Aug. 1999.
[30] O. Edfors, M. Sandell, J.-J. Beek, S. K. Wilson, and P. O. Börjesson, "OFDM channel estimation by singular value decomposition," IEEE Trans. Commun., vol. 46, pp. 931-939, July 1998.

[31] P. Stoica and A. Nehorai, "MUSIC, maximum likelihood, and Cramer-Rao bound," IEEE Trans. Acoust., Speech, Signal Processing, vol. 37, pp. 720-741, May 1989.

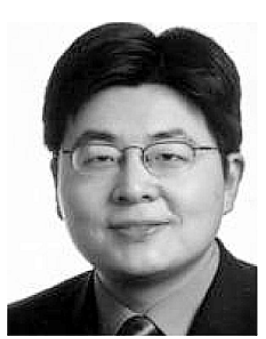

Zhongren Cao (S'01) received the B.Eng. degree from Xi'an JiaoTong University, Xi'an, China, in 1997, and the M.Eng. degree from Shanghai JiaoTong University, Shanghai, China, in 2000, both in electrical engineering. He received the Ph.D. degree in electrical engineering from Stevens Institute of Technology, Hoboken, NJ, in 2004.

Currently, he is a Postdoctoral Researcher with the Department of Electrical and Computer Engineering, University of California, San Diego. His research interests are in the areas of communications, signal processing, networking and software defined radio based system prototype. Recently, his research focuses on broadband multicarrier systems for wireless local area network (WLAN), wireless metropolitan area network (WMAN), and ultra-wideband (UWB) systems, including multiple-access issues, frequency synchronization, channel estimation, MIMO antenna array, and system prototypes.

Dr. Cao has served as Reviewer for various IEEE journals and conferences, including the IEEE TRANSACTIONS ON COMMUNICATIONS, IEEE TRANSACTIONS ON WIRELESS COMMUNICATION, IEEE TRANSACTIONS ON SignAl PROCESSING, ICC, Globecom, VTC, WCNC, etc.

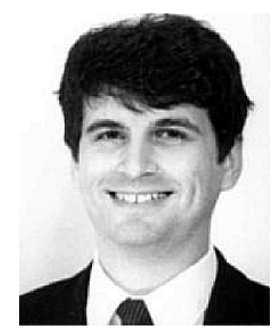

Ufuk Tureli (S'97-M'01) received the B.S. degree in 1994 from Bogazici University, Istanbul, Turkey, and the M.S. and Ph.D. degrees in 1998 and 2000, respectively, from the University of Virginia, Charlottesville, all in electrical engineering.

$\mathrm{He}$ is currently an Assistant Professor at the Department of Electrical and Computer Engineering, Stevens Institute of Technology, Hoboken, NJ. His research interests include diversity and multiplexing for MIMO systems, multiuser communications, and synchronization. He has published numerous journal and conference articles on scalable, adaptive, and robust broadband wireless communications applications.

Dr. Tureli's activities for the IEEE include Reviewer duties for the IEEE TRANSACTIONS ON COMMUNICATIONS and numerous other IEEE journals and conferences. He has served as a chair or on the organizing and technical committees for numerous international conferences. He is a member of the IEEE Communications Society.

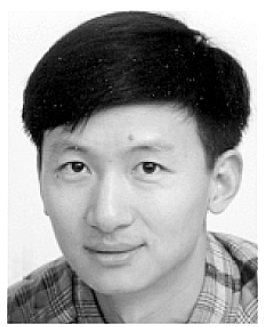

Yu-Dong Yao (S'88-M'88-SM'94) received the B.Eng. and M.Eng. degrees from Nanjing University of Posts and Telecommunications, Nanjing, China, in 1982 and 1985, respectively, and the Ph.D. degree from Southeast University, Nanjing, China, in 1988, all in electrical engineering.

Between 1989 and 1990, he was at Carleton University, Ottawa, ON, Canada, as a Research Associate working on mobile radio communications. He was with Spar Aerospace Ltd., Montreal, QC, Canada, between 1990 and 1994, where he was involved in research on satellite communications. He was with Qualcomm Inc., San Diego, CA, from 1994 to 2000, where he participated in research and development in wireless CDMA systems. He joined Stevens Institute of Technology, Hoboken, $\mathrm{NJ}$, in 2000. He is an Associate Professor in the Department of Electrical and Computer Engineering and a Director of the Wireless Information Systems Engineering Laboratory (WISELAB). He holds one Chinese patent and seven U.S. patents. His research interests include wireless communications and networks, spread spectrum and CDMA, and DSP for wireless systems. He is a Guest Editor for a special issue on wireless networks for the International Journal of Communication Systems.

Dr. Yao is an Associate Editor of IEEE COMMUNICATIONS LETTERS and IEEE TRansactions on Vehicular Technology, and an Editor of IEEE TRANSACTIONS ON WIRELESS COMMUNICATIONS. 